Rev. Int. Contam. Ambie. 35 (Calidad de agua: salud, remediación y perspectiva) 57-64, 2019

DOI: 10.20937/RICA.2019.35.esp03.07

\title{
COMPARACIÓN DE UN BIORREACTOR CON MEMBRANAS SUMERGIDAS CON UN SISTEMA CONVENCIONAL DE LODOS ACTIVADOS PARA EL TRATAMIENTO DE AGUAS RESIDUALES
}

Comparison of a submerged membranes bioreactor with a conventional activated sludge system for wastewater treatment

\author{
Miriam Gabriela FLORES GRANADOS ${ }^{1 *}$, Germán CUEVAS RODRÍGUEZ ${ }^{2}$ \\ y Guillermo GONZALEZ SANCHEZ ${ }^{3}$.
}

${ }^{1}$ Facultad de Ciencias Químicas, Universidad Autónoma de Chihuahia, Circuito Universitario. Chihuahua, México

${ }^{2}$ Departamento de Ingeniería Civil. División de Ingenierías, Campus Guanajuato. Universidad de Guanajuato. Col. Centro. Guanajuato, Guanajuato. CP 36000

${ }^{3}$ Centro de Investigación en Materiales Avanzados, CIMAV. Miguel de Cervantes 120, Complejo Industrial Chihuahua Chihuahua, Chih. México. C.P. 31136

*Autor de correspondencia: mgfloresg@uach.mx

(Recibido febrero 2019, aceptado abril 2019)

Palabras clave: tratamiento de aguas, BRMS, eficiencia

\section{RESUMEN}

El tratamiento de aguas residuales se ha incrementado en las últimas décadas. El objetivo de este estudio fue comparar dos sistemas para el tratamiento de aguas residuales. El primer sistema fue el biorreactor con membrana sumergida (BRMS) y el segundo fue el sistema convencional de lodos activados (RLA). Los parámetros evaluados fueron demanda química de oxígeno total ( $\left.\mathrm{DQO}_{\text {total }}\right)$, demanda química de oxígeno soluble $\left(\mathrm{DQO}_{\text {soluble }}\right)$, fosfatos $\left(\mathrm{PO}_{4}{ }^{3-}\right)$, amoniaco $\left(\mathrm{NH}_{4}{ }^{+}\right)$, nitratos $\left(\mathrm{NO}_{3}{ }^{-}\right)$, sólidos suspendidos totales (SST), sólidos suspendidos volátiles (SSV) y $\mathrm{pH}$, y se determinaron en muestras obtenidas de la alimentación y del efluente de los sistemas tres veces por semana. Se preparó diariamente el agua residual sintética que fue usada en los dos reactores para evitar la degradación en el tanque de almacenamiento. Los resultados, como la DQOTotal, muestran que hay una mejoría usando el BRMS con respecto al RLA. Además, la filtración que se lleva a cabo permite una remoción mayor de SST y el proceso de nitrificación en el primer sistema se llevó a cabo convirtiendo el $\mathrm{NH}_{4}^{+}$en $\mathrm{NO}_{3}{ }^{-}$. Se concluye que el efluente del BRMS es de mejor calidad debido a que tiene menos contaminantes, por lo que ese sistema puede ser utilizado para el tratamiento de aguas residuales.

Key words: water treatment, SMBR, efficiency

\begin{abstract}
Wastewater treatment has increased in recent decades. The objective of this study was to compare two systems for wastewater treatment. The first system was the submerged membrane bioreactor (SMBR) and the second was the conventional system of activated sludge (RLA). The parameters evaluated were total chemical oxygen demand $\left(\mathrm{COD}_{\text {to- }}\right.$ tal), soluble chemical oxygen demand $\left(\mathrm{COD}_{\text {soluble }}\right)$, phosphates $\left(\mathrm{PO}_{4}{ }^{3-}\right)$, nitrates $\left(\mathrm{NH}_{4}^{+}\right)$,
\end{abstract}


nitrites $\left(\mathrm{NO}_{3}^{-}\right)$, total suspended solids (TSS), volatile suspended solids (VSS) and $\mathrm{pH}$, and were determined in samples obtained from the feeding and the effluent of the systems three times a week. The wastewater used in the two reactors was prepared daily to

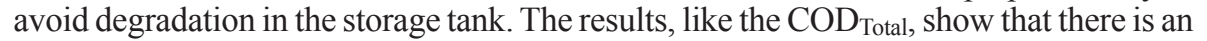
improvement using the SMBR with respect to the RLA. Besides, the filtration carried out allowed a greater removal of TSS and the nitrification process in the SMBR converted $\mathrm{NH}_{4}{ }^{+}$in $\mathrm{NO}_{3}^{-}$. It is concluded that the effluent of the SMBR is of better quality because it had less contaminants and it can be used for wastewater treatment.

\section{INTRODUCCIÓN}

Uno de los grandes problemas que enfrentan en la actualidad las poblaciones urbanas es la disponibilidad de agua. Debido a eso se hace necesario su ahorro y una mejor distribución, así como la reutilización del agua tratada que pueda ser usada en distintas áreas, como la agricultura y en otros sectores. El procedimiento más utilizado para tratar las aguas residuales es el de lodos activados (RLA) al cual también se le conoce como tratamiento secundario. El proceso de RLA consiste en un tratamiento biológico en donde la materia orgánica es degradada por microorganismos aerobios al estar ambos en contacto bajo condiciones óptimas de aireación. Un proceso de RLA requiere de un reactor llamado tanque de aireación, un sedimentador secundario y un sistema de recirculación de lodos (Rittmann y McCarty 2001).

Una de las modificaciones en el proceso de tratamiento biológico convencional es el reemplazo del sedimentador secundario por unidades de membranas, el cual se conoce como biorreactor con membranas (BRM). Este proceso está emergiendo como una tecnología con ventajas considerables sobre los métodos de tratamiento convencionales (Lee et al. 2001, Cornelissen et al. 2002, Merlo et al. 2004, Yoon et al. 2004). Los bioreactores con membranas (BRM) se pueden definir como la combinación de dos procesos básicos: la degradación biológica y la separación por membrana. Al implementar este proceso, los sólidos y los microorganismos son separados del agua tratada mediante una unidad de filtración. Debido a que la biomasa se queda dentro del reactor, ésta proporciona un control del tiempo de residencia de los microorganismos en el reactor y la desinfección del efluente (AWWARF 1999, Ahn y Song 1999, Innocenti et al. 2002, Sofia et al. 2004, Melin et al. 2006).

En la actualidad existen dos configuraciones de biorreactores con membranas: los de membrana sumergida y los de membranas externas. En los biorreactores con membrana sumergida o integrada, la filtración se realiza sumergida en el biorreactor. De esta manera, los lodos quedan dentro del reactor y el efluente es retirado (Fig. 1a). En los biorreactores con membrana externa el licor de mezcla es recirculado a la unidad de filtración con el apoyo de una bomba y se regresan los lodos (Fig. 1b) (AWWARF 1999, Till y Mallia 2001, Tazi-Pain et al. 2002, Merlo et al. 2004).

En comparación con el proceso de lodos activados, el biorreactor con membrana sumergida tiene las ventajas de excelente calidad de efluente, retención de todos los sólidos suspendidos y microorganismos, control absoluto de la biomasa y del tiempo de retención hidráulico, entre otras (Massé et al. 2006, Melin et al. 2006). Varios estudios han demostrado que este sistema es efectivo para la eliminación de nitrógeno y el rendimiento en general es mejor que el del proceso de tratamiento convencional (Gao et al. 2004, Laera et al. 2005).
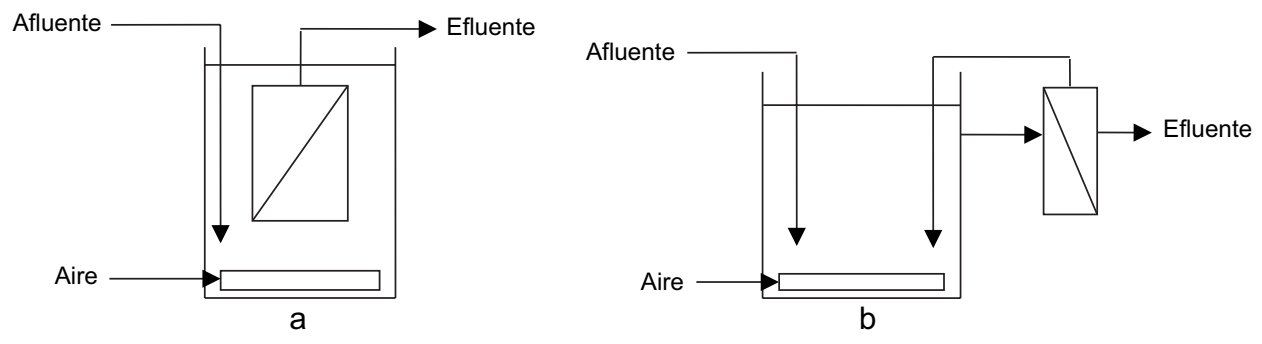

Fig. 1. Biorreactor con membrana sumergida (a) y biorreactor con membrana externa (b) (Melin et al. 2006, AWWARF 1999) 
El aumento del consumo de agua implica que se deban buscar nuevas tecnologías que permitan su reutilización. En México el sistema de tratamiento de aguas residuales que más se emplea es el de lodos activados. Este sistema no es eficiente, ocupa mucho espacio y genera lodos que deben ser tratados para su uso o disposición final y el agua tratada que se obtiene se puede usar en algunas actividades como riego de parques. Si se implementara tecnología avanzada como el reactor con membranas, al agua tratada se le podría dar un uso más amplio como en la industria, la construcción, etc., además de reducir el impacto ambiental en los cuerpos receptores. Esta tecnología ocupa menos espacio y el sistema de filtrado puede usar membranas de distintos materiales y tamaños de poro disponibles en el mercado. El objetivo del trabajo fue comparar la eficiencia de dos configuraciones de reactores biológicos aerobios (reactor de lodos activados convencional y reactor biológico con membranas sumergidas), para llevar a cabo el tratamiento de aguas residuales domésticas.

\section{MATERIALES Y MÉTODOS}

El estudio se desarrolló utilizando dos reactores piloto: el bioreactor con membranas sumergidas (BRMS) y el reactor convencional de lodos activados (RLA). Los sistemas se instalaron en el Centro de Investigación en Materiales Avanzados (CIMAV) y se evaluaron durante un periodo de 40 días. El BRMS (Fig. 2) tuvo un volumen neto de $32.5 \mathrm{~L}$ y dos módulos de membranas (aireación y filtración). El módulo de aireación sirvió para proporcionar el oxígeno a los microorganismos y poder llevar a cabo la mezcla del reactor. Las características de las membranas de este módulo son las siguientes: planas, hidrofóbicas, de ultrafiltración UltraPes ${ }^{\circledR}$, de $0.1 \mathrm{~mm}$ de tamaño de poro y un área superficial total de $0.2016 \mathrm{~m}^{2}$. Por otro lado, las características del módulo de filtración fueron las siguientes: membranas planas (Milipore), hidrofílicas, de microfiltración ( $0.45 \mathrm{~mm}$ de tamaño de poro) y un área superficial total de $0.14 \mathrm{~m}^{2}$.

El RLA estuvo compuesto por un reactor aerobio y un sedimentador, en los que el volumen fue de 39 L y de 5.6 L, respectivamente, con recirculación de lodos (Fig. 3). La aireación se llevó a cabo a través de un difusor colocado al fondo del reactor, el cual tenía las siguientes características: perforaciones de 1/8 de pulgada, forrado con fibra para obtener un menor tamaño de burbuja y favorecer el mezclado dentro del reactor. El sedimentador fue circular, donde los lodos sedimentaban por gravedad.

\section{Condiciones de operación}

Las condiciones de operación que se manejaron en los sistemas se muestran en el Cuadro I. Uno de los principales parámetros en la operación fue el valor de carga orgánica aplicada, la cual fue de 0.264 para el BRMS y de $0.844 \mathrm{~kg}$ DQO Total $_{1} / \mathrm{kg} \mathrm{SST} / \mathrm{d}$ para el RLA. La concentración media de sólidos suspendidos totales en licor de mezcla (SSTLM) fue muy similar en ambos reactores (1202.4 y $1162.5 \mathrm{mg} / \mathrm{L}$, respectivamente). En el BRMS no existió una purga de lodos durante el estudio por lo que el tiempo de retención celular (TRC) fue de $40 \mathrm{~d}$ y en el RLA, la recirculación de lodos fue de $160 \mathrm{~mL} / \mathrm{min}$ con un TRC de $8 \mathrm{~d}$. El tiempo de retención hidráulica (TRH) en el BRMS fue de $73.4 \mathrm{~h}$ mientras que en el RLA fue de $16.6 \mathrm{~h}$.

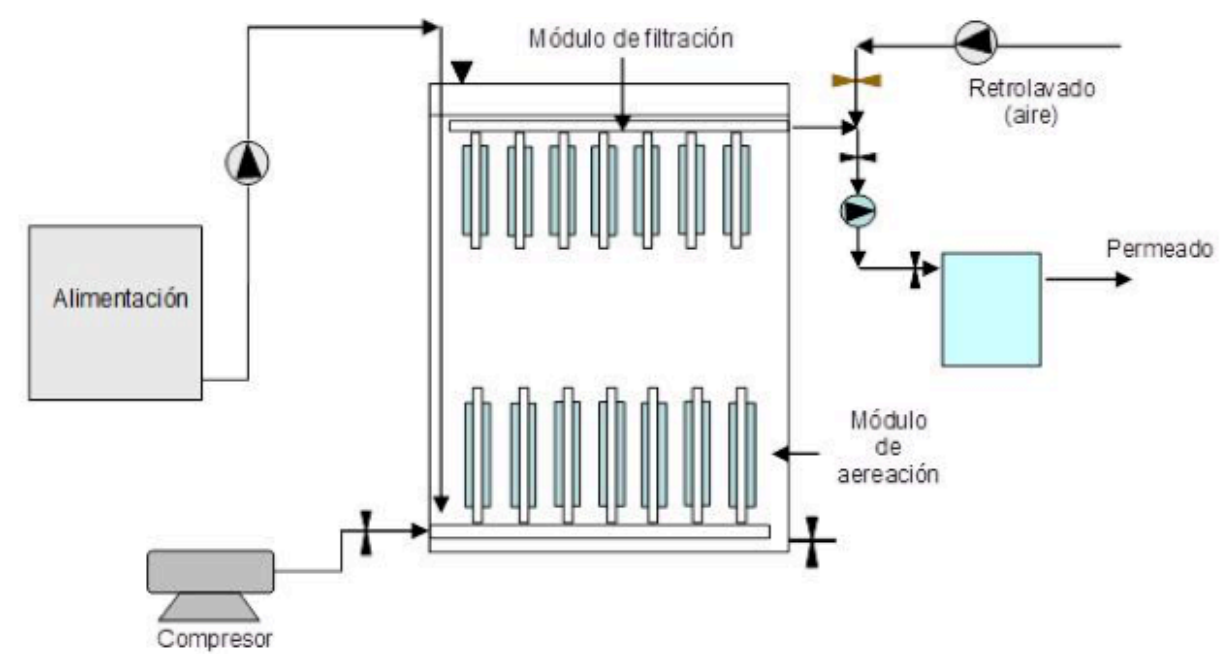

Fig. 2. Biorreactor con membranas sumergidas 


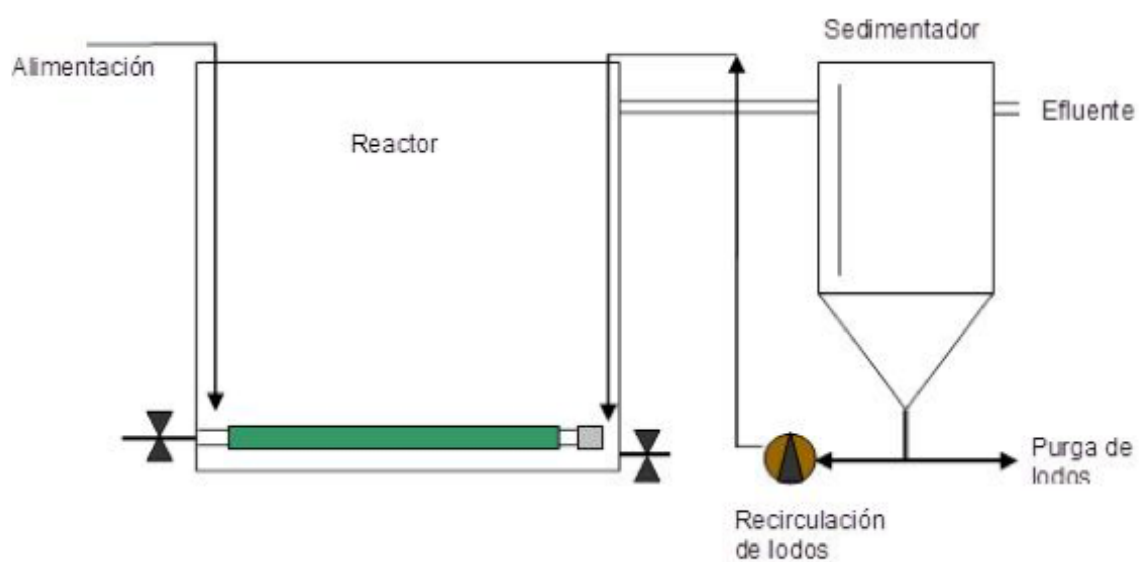

Fig. 3. Sistema de lodos activados

CUADRO I. CONDICIONES DE OPERACIÓN MANEJADAS EN EL BRMS Y EN EL RLA

\begin{tabular}{lccc}
\hline Parámetro & Unidad & BRMS Valor & RLA Valor \\
\hline Caudal (Q) & $\mathrm{L} / \mathrm{h}$ & 0.44 & 2.34 \\
Carga orgánica (CO) & $\mathrm{kg} \mathrm{DQO}$ Total $/ \mathrm{kg} \mathrm{SST} * \mathrm{~d}^{(+)}$ & 0.264 & 0.844 \\
$\begin{array}{l}\text { Solidos suspendidos totales } \\
\text { en licor mezcla (SSTLM) }\end{array}$ & $\mathrm{mg} / \mathrm{L}$ & 1202.4 & 1162.5 \\
Solidos suspendidos volátiles en & & & \\
licor mezcla (SSVLM) & $\mathrm{mg} / \mathrm{L}$ & 1012.8 & 1077.8 \\
pH & - & 7.06 & 7.25 \\
Oxígeno disuelto (OD) & $\mathrm{mg} / \mathrm{L}$ & 2.52 & 1.90 \\
Temperatura & ${ }^{\circ} \mathrm{C}$ & 26.70 & 26.9 \\
\hline
\end{tabular}

${ }^{(+)}$Kilogramo de $\mathrm{DQO}_{\text {total }} /$ kilogramo solidos suspendidos totales por día

\section{Características de las aguas residuales}

Los dos reactores se alimentaron con agua residual sintética utilizando la formulación modificada de Holler y Trösch (2001) cuya composición se muestra en el Cuadro II. El agua se preparó diariamente con el propósito de evitar su degradación en el tanque de almacenamiento. En el Cuadro III se muestran los parámetros de calidad del agua residual sintética

CUADRO II. COMPOSICIÓN MEDIA DEL AGUA RESIDUAL SINTÉTICA

\begin{tabular}{lr}
\hline Compuesto & $\mathrm{mg} / \mathrm{L}$ \\
\hline Dextrosa monohidratada & 446.107 \\
Extracto de carne & 237.725 \\
$\mathrm{Urea}$ & 129.670 \\
$\mathrm{~K}_{2} \mathrm{HPO}_{4}$ & 121.025 \\
$\mathrm{NaCl}_{\mathrm{CaCl}} \cdot \mathrm{H}_{2} \mathrm{O}$ & 15.130 \\
$\mathrm{MgSO}_{4} \cdot 7 \mathrm{H}_{2} \mathrm{O}$ & 8.645 \\
\hline
\end{tabular}

CUADRO III. PARÁMETROS DE CALIDAD DEL AGUA DE ALIMENTACIÓN DE LOS REACTORES

\begin{tabular}{lcccc}
\hline Parámetro & $\begin{array}{c}\text { Valor } \\
\text { promedio }\end{array}$ & $\begin{array}{c}\text { Unidad } \\
\text { Std }\end{array}$ & Método \\
\hline DQO $_{\text {Total }}$ & 681 & $\mathrm{mg} / \mathrm{L}$ & \pm 180.71 & 8000, Hach \\
DQOSoluble & 538 & $\mathrm{mg} / \mathrm{L}$ & \pm 167.94 & 8000, Hach \\
$\mathrm{SST}$ & 71 & $\mathrm{mg} / \mathrm{L}$ & \pm 35.88 & $2540 \mathrm{D}$, APHA \\
$\mathrm{SSV}$ & 67 & $\mathrm{mg} / \mathrm{L}$ & \pm 35.01 & $2540 \mathrm{E}, \mathrm{APHA}$ \\
$\mathrm{NH}_{3}{ }^{-}$ & 20 & $\mathrm{mg} / \mathrm{L}$ & \pm 14.12 & 8039, Hach \\
$\mathrm{PO}_{4}{ }^{3-}$ & 76 & $\mathrm{mg} / \mathrm{L}$ & \pm 20.05 & 8114, Hach \\
pH & 6.68 & - & \pm 20.05 & $4500-\mathrm{H}^{+} \mathrm{B}, \mathrm{APHA}$ \\
Temperatura & 28 & ${ }^{\circ} \mathrm{C}$ & \pm 2.2 & 2550, APHA \\
\hline
\end{tabular}

usada en este estudio, los cuales son representativos de las aguas residuales domésticas.

\section{Métodos analíticos}

La determinación de parámetros en la alimentación y en el efluente de los reactores se midió tres 
veces por semana. Las determinaciones se realizaron en muestras compuestas, las cuales fueron analizadas en el Laboratorio de Control de Agua del CIMAV. Los análisis que se realizaron para cuantificar el contenido total, orgánico e inorgánico de la biomasa suspendida fueron: sólidos suspendidos totales (SST) y sólidos suspendidos volátiles (SSV). Estos se realizaron de acuerdo con lo descrito por Standard Methods (APHA 2005). La demanda química de oxígeno total $\left(\mathrm{DQO}_{\text {Total }}\right)$ y soluble (DQO $\left.{ }_{\text {Soluble }}\right)$, el nitrógeno amoniacal $\left(\mathrm{N}-\mathrm{NH}_{3}{ }^{-}\right)$y los fosfatos $\left(\mathrm{P}-\mathrm{PO}_{4}{ }^{3-}\right)$ fueron analizados por los métodos de Hach (Hach 2005)..

Algunos de los parámetros de operación de ambos reactores fueron determinados con equipos portátiles de campo. La medición del oxígeno disuelto (OD) se realizó mediante un oxímetro de la marca Hach modelo Ion 5, mientras que el monitoreo del $\mathrm{pH}$ y la temperatura se realizó con el medidor multiparámetro Orion 4-star plus de Thermo Scientific.

\section{RESULTADOS}

\section{Sólidos suspendidos (SS)}

La concentración promedio de SST en la alimentación fue de $71 \mathrm{mg} / \mathrm{L}$ y de SSV de $67 \mathrm{mg} / \mathrm{L}$. El porcentaje de remoción de SST en el efluente del BRMS fue de $89.71 \%$ mientras que en el efluente del RLA fue de $54.41 \%$. Para el caso de los SSV, los valores fueron $85.60 \%$ y $56.16 \%$ respectivamente. En las figuras $\mathbf{4}$ y $\mathbf{5}$ se observa cómo disminuye la concentración de SST y SSV en el efluente del BRMS debido a las membranas de microfiltración (MF) y que se obtiene un filtrado sin sólidos. En el caso del RLA, el comportamiento de las concentraciones de los SST y SSV en el efluente indica que la eficiencia de remoción de los sólidos suspendidos se llevó a cabo correctamente, sin embargo, en el BRMS la efi-

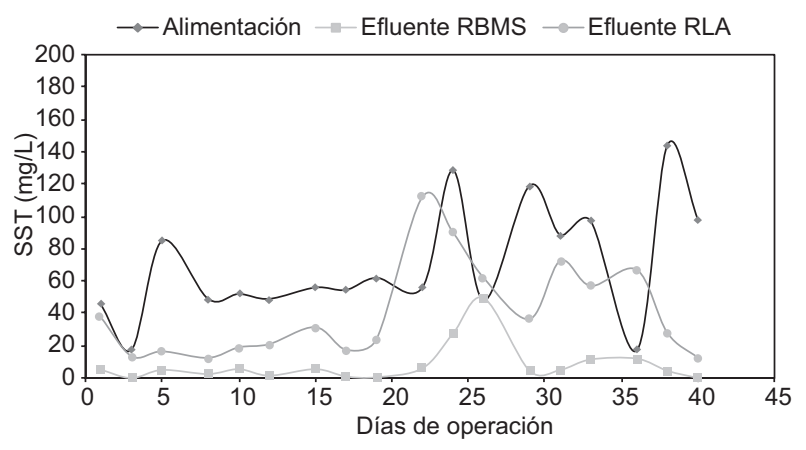

Fig. 4. Comportamiento de los SST en el BRMS y en el RLA durante el estudio

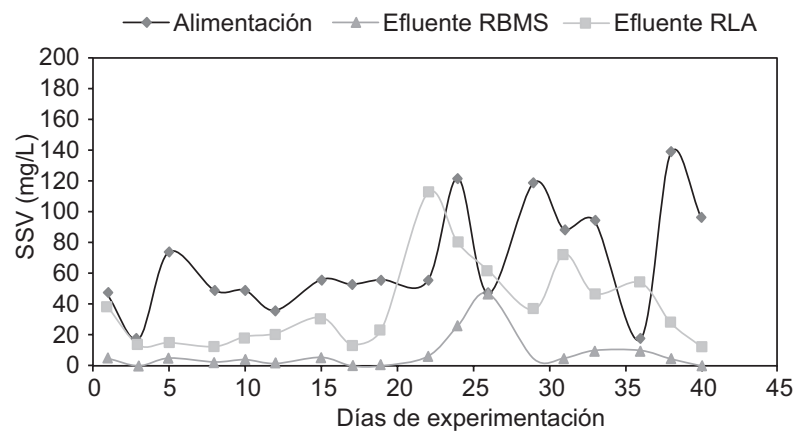

Fig. 5. Comportamiento de los SSV en el BRMS y en el RLA durante el estudio

ciencia de remoción alcanzada, durante el tiempo que duró la experimentación, fue mayor.. Para efluentes de BRMS varios autores reportan eficiencias arriba del $99 \%$ de SST y SSV e incluso casos sin detección de ellos (Shim et al. 2002, Laera et al. 2005). Los porcentajes de remoción mostrados anteriormente están por debajo de lo reportado en otros estudios. En este estudio, los resultados muestran que en el BRMS se obtuvo un mejor rendimiento que en el RLA.

\section{Demanda química de oxígeno (DQO)}

La concentración media de DQOTotal en la alimentación fue de $681.4 \mathrm{mg} / \mathrm{L}$ mientras que en el efluente del BRMS la concentración promedio de $\mathrm{DQO}_{\text {Total }}$ fue de $85.1 \mathrm{mg} / \mathrm{L}$ y en el RLA fue de $117.7 \mathrm{mg} / \mathrm{L}$. El porcentaje de remoción de $\mathrm{DQO}$ Total $\mathrm{y}$ de $\mathrm{DQO}$ Soluble para el efluente del BRMS fue de $87.52 \%$ y $84.91 \%$, respectivamente; mientras que para el RLA los resultados fueron $81.85 \%$ y $85.68 \%$, respectivamente. En el Cuadro IV se encuentran las concentraciones de DQOTotal y de DQOSoluble en la alimentación y en los efluentes de los reactores.

CUADRO IV. CONCENTRACIONES DE DQO Total Y DQO Soluble

\begin{tabular}{lcc}
\hline Muestra & DQO $_{\text {Total }}(\mathrm{mg} / \mathrm{L})$ & DQO $_{\text {Soluble }}(\mathrm{mg} / \mathrm{L})$ \\
\hline Alimentación & 681.4 & 537.5 \\
Efluente BRMS & 85.1 & 76.2 \\
Efluente RLA & 117.7 & 70.9 \\
\hline
\end{tabular}

Se ha reportado que para un efluente de BRMS los porcentajes de remoción de $\mathrm{DQO}_{\text {Total }} \mathrm{y}$ de $\mathrm{DQO}$ Solubre obtenidos fueron $90 \%$ a $98 \%$ (para ambos parámetros), para un efluente de RLA los valores fueron $77.5 \%$ a $93.8 \%$ y de $87.4 \%$ a $97 \%$, respectivamente (Ng y Hermanowicz 2005, Massé et al. 2006). 
Los resultados obtenidos en este trabajo experimental muestran que los porcentajes de remoción de DQO (total y soluble) son más bajos que los reportados en la literatura; haciendo un análisis de la información se observa que el porcentaje de remoción de DQOTotal alcanzando en el BRMS fue $4.78 \%$ más que en el RLA, mientras que la remoción de $\mathrm{DQO}$ soluble fue $0.98 \%$ más alta en el RLA que en el RBMS.

\section{Amonio y nitratos $\left(\mathrm{NH}_{4}{ }^{+}, \mathrm{NO}_{3}{ }^{-}\right)$}

La concentración de $\mathrm{NH}_{4}{ }^{+}$en la alimentación fue de19.9 mg/L, en el efluente del BRMS fue de $14.3 \mathrm{mg} / \mathrm{L}$ y en el efluente del RLA de $25.5 \mathrm{mg} / \mathrm{L}$ (Cuadro V). En la figura 6, en la alimentación la concentración estuvo variando, este efecto posiblemente se explique por la falta de agitación continua en el tanque de alimentación. En el efluente del BRMS la concentración disminuyó debido al proceso de nitrificación que se llevó a cabo. La concentración en el efluente del RLA se observa con variaciones significativas debida probablemente a las variaciones de la concentración de amonio en la alimentación (Cuadro V y Fig. 6). En este tipo de reactor las bacterias nitrificantes tardaron más en crecer debido a que los lodos no estuvieron confinados como en el BRMS, donde los tiempos de retención celular (TRC) fueron más grandes que en los sistemas convencionales. Esto significa que para poder remover

CUADRO V. CONCENTRACIONES DEAMONIO, NITRATOS Y FOSFORO EN LAALIMENTACIÓN Y EN LOS EFLUENTES

\begin{tabular}{lccc}
\hline & $\begin{array}{c}\mathrm{NH}_{4}{ }^{+} \\
(\mathrm{mg} / \mathrm{L})\end{array}$ & $\begin{array}{c}\mathrm{NO}_{3}{ }^{-} \\
(\mathrm{mg} / \mathrm{L})\end{array}$ & $\begin{array}{c}\mathrm{PO}_{4}{ }^{3-} \\
(\mathrm{mg} / \mathrm{L})\end{array}$ \\
\hline Alimentación & 19.9 & - & 75.5 \\
Efluente BRMS & 14.3 & 33.9 & 82.2 \\
Efluente RLA & 25.5 & 25.1 & 74.3 \\
\hline
\end{tabular}

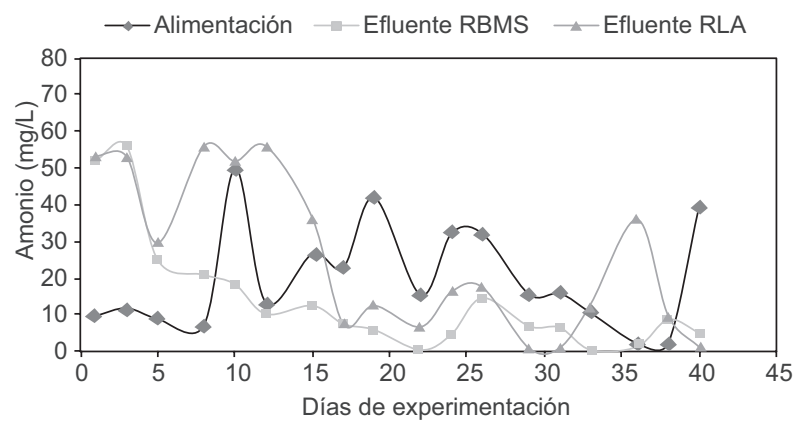

Fig. 6. Concentraciones de amonio en la alimentación y en los efluentes de los reactores amonio con bacterias nitrificantes se deben tener TRC mayores a $5 \mathrm{~d}$, de acuerdo con la figura 7 en donde se ve que en el BRMS ese compuesto empieza a disminuir. En el caso del RLA, se requiere de más tiempo para que ocurra la remoción y se realizan purgas de lodos para mantener una cantidad de sólidos constante en el reactor.

En el caso del $\mathrm{NO}_{3}{ }^{-}$, los datos obtenidos en el efluente del BRMS y en el efluente del RLA fueron $33.89 \mathrm{mg} / \mathrm{L}$ y $25.07 \mathrm{mg} / \mathrm{L}$, respectivamente (Cuadro V) y su presencia significa que se está llevando la nitrificación en ambos reactores (Fig. 7). Los nitratos en el efluente del BRMS muestran que durante el estudio las concentraciones se mantuvieron altas debido a la conversión del $\mathrm{NH}_{4}{ }^{+}$a $\mathrm{NO}_{3}{ }^{+}$. En la figura 7 se observa la presencia de nitratos en el efluente de RLA indicando que se llevó cabo la nitrificación en el reactor de lodos activados.

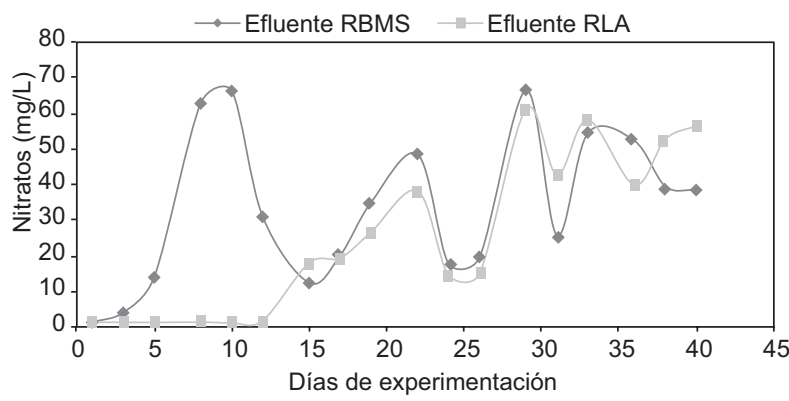

Fig. 7. Concentraciones de nitratos en los efluentes de los reactores

Se han reportado concentraciones obtenidas de $\mathrm{NO}_{3}{ }^{-}$de $13.5 \mathrm{mg} / \mathrm{L}$ a $25.4 \mathrm{mg} / \mathrm{L}$ en efluentes de BRMS y para efluentes de RLA valores desde $3 \mathrm{mg} / \mathrm{L}$ hasta $20 \mathrm{mg} / \mathrm{L}$, usando agua residual sintética (Hasar y Kinacy 2004, Ng y Hermanowicz 2005). Otros autores (Shim et al. 2002) obtuvieron una eficiencia de nitrificación alrededor del 95\%, la que resultó de altos TRC y altas concentraciones de lodos empleando un reactor con membranas planas. En este estudio se usó un TRC de $40 \mathrm{~d}$ en donde se observó que el proceso de nitrificación en el BRMS tuvo un mejor resultado que en el RLA, pudiéndose mejorar al aumentar el tiempo de operación del reactor.

\section{Fosfatos $\left(\mathrm{PO}_{4}{ }^{3-}\right)$}

En el Cuadro V se especifican las concentraciones promedio de $\mathrm{PO}_{4}{ }^{3-}$ en la alimentación y en los efluentes de los reactores. La concentración de fosfatos en el efluente del BRMS muestra que hubo acumulación de este macronutriente durante el 


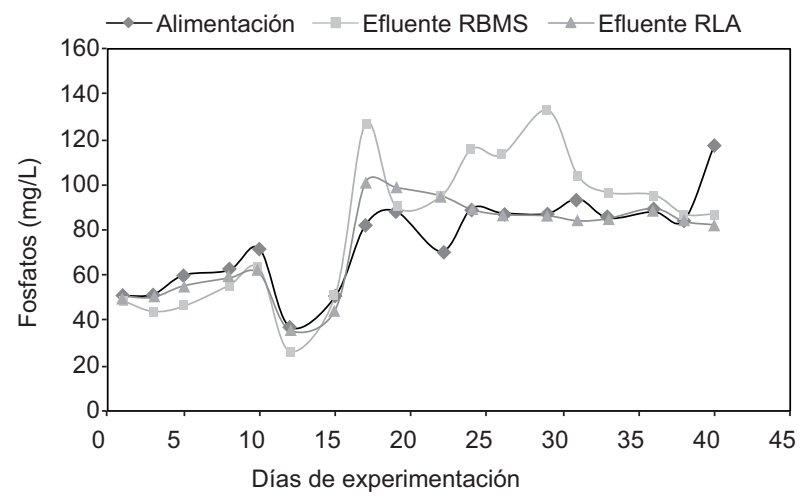

Fig. 8. Concentraciones de $\mathrm{PO}_{4}{ }^{3-}$ en la alimentación y en los efluentes de los reactores

periodo de experimentación debido al largo tiempo de retención de los lodos en el reactor. Como consecuencia de este efecto, posiblemente el $\mathrm{PO}_{4}{ }^{3-}$ asimilado por los microorganismos se fue acumulando junto con el suministrado en la alimentación. Por otro lado, se observó que en el RLA la concentración de $\mathrm{PO}_{4}{ }^{3-}$ en efluente presentó valores muy similares a los detectados en agua residual de la alimentación, esto indica que al interior del reactor no había condiciones adecuadas para llevar a cabo la remoción de fosfatos (Fig. 8).

Algunos estudios indican que existen porcentajes de eliminación de $\mathrm{PO}_{4}{ }^{3-}$ que van del $11 \%$ hasta el $75 \%$ (Stephenson et al. 2000). Otros autores reportan eficiencias de remoción de $\mathrm{PO}_{4}{ }^{3-}$ para BRMS de $81.9 \%$ y para el RLA de $32.4 \%$ (Hasar y Kinaci 2004). De acuerdo con las referencias, puede haber buena remoción de $\mathrm{PO}_{4}{ }^{3-}$ en un BRMS pero se requieren de TRC mayores al que se manejó en este estudio que fue de $40 \mathrm{~d}$ y la presencia de condiciones anóxicas. En el caso del RLA, la remoción fue baja y se requiere de un tratamiento adicional para lograr una remoción más significativa de fosfatos.

\section{pH}

En la alimentación el promedio del $\mathrm{pH}$ fue de 6.66. Este parámetro en ambos efluentes se mostró

CUADRO VI. VALORES DE PH PROMEDIO OBTENIDOS DURANTE EL ESTUDIO

\begin{tabular}{lc}
\hline Muestra & $\mathrm{pH}$ promedio \\
\hline Alimentación & 6.68 \\
Efluente BRMS & 7.29 \\
Efluente RLA & 7.36 \\
BRMS & 7.06 \\
RLA & 7.25 \\
\hline
\end{tabular}

muy similar ya que en el efluente del BRMS el promedio fue $7.77 \mathrm{y}$ en el efluente del RLA fue de 7.80 (Cuadro VI). El pH es un parámetro que debe ser bien controlado en los sistemas de tratamiento. Es importante mantenerlo en un rango de 6.5 a 8.5 para evitar la formación de microorganismos no deseados en los reactores como bacterias filamentosas que provocan problemas como mala sedimentación de los lodos y alta cantidad de sólidos en los efluentes que se traduce en un agua tratada de mala calidad (Metcalf y Eddy 2003).

\section{CONCLUSIONES}

Los resultados obtenidos de las concentraciones de DQO (total y soluble) y SS (totales y volátiles) en los efluentes del BRMS son menores que los obtenidos en la literatura, sin embargo indican una mayor remoción con respecto de los valores obtenidos del efluente del RLA. La concentración de nitratos en el efluente del BRMS fue más alta que en el efluente del RLA debido al proceso de nitrificación que se presentó al manejar un TRC de $40 \mathrm{~d}$ en el BRMS, lo que favoreció una reducción en la concentración del amonio. Para que los fosfatos sean removidos del efluente de BRMS se requiere de un proceso anóxico para favorecer la asimilación de fosforo por las bacterias y así removerlo del agua. Los resultados que se obtuvieron con el BRMS muestran que es una tecnología que se puede usar para el tratamiento y reuso de agua residual tratada.

\section{AGRADECIMIENTOS}

Se agradece al Centro de Investigación en Materiales Avanzados (CIMAV) por permitir el uso de sus instalaciones y equipo, así como al personal técnico de Laboratorio de Calidad de Agua.

\section{REFERENCIAS}

Ahn K.H., Song K.G. (1999). Treatment of domestic wastewater using microfiltration for the reuse of wastewater. Desalination 126: 4-14. DOI: 10.1016/S0011-9164(99)00150-2

American Water Works Association Research Foundation (AWWARF), Lyonnaise des Eaux, Water Research Commission of South Africa. (1999). Tipos de operaciones de membranas. J. Mallevialle, P.E. Odendaal, M.R. Wiesner. Tratamiento del agua por procesos de 
membrana. Mc Graw Hill. España, pp: 13-39.

APHA. (2005). Standard Methods for the Examination of Water and Wastewater. American Public Health Association/American Water Works Association/Water Environment Federation. 21 ed. Port City Press, Baltimore, Maryland.

Cornelissen E.R., Janse W., y Koning J. (2002). Wastewater treatment with the internal MEMBIOR. Desalination 146, 463-466. DOI: 10.1016/S0011-9164(02)00549-0

Cuevas-Rodríguez G, Flores-Granados M, GonzálezSánchez G, Nevarez-Moorillon G V. Aplicación de un biorreactor con membranas sumergidas para el tratamiento de aguas residuales. XXXI Congreso Interamericano AIDIS. (2008). 12-15 Octubre. Santiago, Chile.

Gao M., Yang M., Li, H., Wang Y. y Pan F. (2004). Nitrification and sludge characteristics in a submerged membrane bioreactor on synthetic inorganic wastewater. Desalination 170: 177-185.

DOI: 10.1016/j.desal.2004.02.099

Hach (2005). Métodos, manual de análisis de agua. Traducción al español de la 2 da edición de Water analysis handbook. Hach Company, EUA.

Hasar H. y Kinaci C. (2004) Comparison of a sMBR and a CASP system for wastewater reclamation and reuse. Filtration and Separation 41: 35-39.

DOI: $10.1016 / \mathrm{S} 0015-1882(04) 00111-9$

Holler S. y Trösch W. (2001). Treatment of urban wastewater in a membrane bioreactor at high organic loading rates. Journal of Biotechnology 92: 95-101.

DOI: 10.1016/S0168-1656(01)00351-0

Innocenti L., Bolzonella D., Pavan P. y Cecchi F. (2002). Effect of sludge age on the performance of a membrane bioreactor: influence on nutrient and metals removal. Desalination 146: 467-474.

DOI: 10.1016/S0011-9164(02)00551-9

Laera G., Pollice A., Saturno D., Giordano C. y López A. (2005). Zero net growth in a membrane bioreactor with complete sludge retention. Water Research 39: 52415249. DOI: 10.1016/j.watres.2005.10.010

Lee J., Ahn W-Y. y Lee C-H. (2001). Comparison of the filtration characteristics between attached and suspended growth microorganisms in submerged membrane bioreactor. Water Research 35 (10), 2435-2445.

DOI: $10.1016 / \mathrm{S} 0043-1354(00) 00524-8$

Massé A., Spérandio M. y Cabassud C. (2006). Comparison of sludge characteristics and performance of a submerged membrane bioreactor and an activated sludge process at high solids retention time. Water Research 40: 2405-2415.

DOI: $10.1016 /$ j.watres.2006.04.015
Metcalf y Eddy. (2003). Fundamentals of biological treatment. Wastewater engineering: treatment and reuse. Mc Graw Hill, pp: 695-700.

Melin T., Jefferson B., Bixio D., Thoeye C., De Wilde W., De Koning J., Van der Graaf J., y Wintgens T. (2006). Membrane bioreactor technology for wastewater treatment and reuse. Desalination 187: 271-282.

DOI: 10.1016/j.desal.2005.04.086

Merlo R.P., Trussell S., Hermanowicz S.W. y Jenkins D. (2004). Physical, chemical and biological properties of submerged membrane bioreactor and conventional activated sludges. Water Environmental Federation, Alexandria, Virginia, EUA.

Ng H.Y., y Hermanowicz S.W. (2005). Membrane bioreactor operation at short solids retention times: performance and biomass characteristics. Water Research 39, 981-992. DOI: 10.1016/j.watres.2004.12.014

Rittman B.E., McCarty P.L. (2001). The activated sludge process. Environmental Biotechnology: Principles and Applications. Mc Graw Hill. Nueva York., pp: 307-395.

Shim J.K., Yoo I.-K. y Lee Y. M. (2002). Design and operation considerations for wastewater treatment using a flat submerged membrane bioreactor. Process Biochemistry 38: 279-285.

DOI: 10.1016/S0032-9592(02)00077-8

Sofia A., Ng W.J., Ong S.L. (2004). Engineering design approaches for minimum fouling in submerged MBR. Desalination 160: 67-74. DOI: 10.1016/S0011-9164(04)90018-5

Stephenson T., Judd S., Jefferson B. y Brindle K. (2000). Biomass separation membrane bioreactors. Membrane bioreactors for wastewater treatment. IWA. Londres, Reino Unido, pp: 59-111

Tazi-Pain A., Schrotter J.C., Bord G., Payreaudeau M., y Buisson H. (2002). Recent improvement of the BIOSEP ${ }^{\circledR}$ process for industrial and municipal wastewater treatment. Desalination 146: 439-443.

DOI: 10.1016/S0011-9164(02)00538-6

Till S. y Mallia H. (2001). Membrane bioreactors: wastewater treatment applications to achieve high quality effluent. $64^{\text {th }}$ Annual Water Industry Engineers and Operators' Conference. Bendigo, Australia.

Yoon S.H., Kim H.S. y Yeom I.T. (2004). The optimum operational condition of membrane bioreactor (MBR): cost estimation of aeration and sludge treatment. Water Research 38, 37-46.

DOI: 10.1016/j.watres.2003.09.001 